Article

\title{
Study on Droplet Size and Velocity Distributions of a Pressure Swirl Atomizer Based on the Maximum Entropy Formalism
}

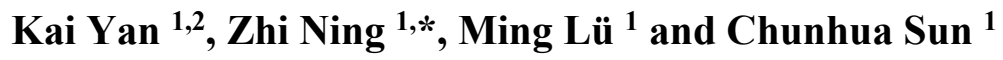

1 School of Mechanical, Electronic and Control Engineering, Beijing Jiaotong University, Beijing 100044, China; E-Mails: yanqiaokai@gmail.com (K.Y.); 08121920@bjtu.edu.cn (M.L.); jasonshangko@hotmail.com (C.S.)

2 Institute of Electrical Engineering, Chinese Academy of Sciences, Beijing 100190, China

* Author to whom correspondence should be addressed; E-Mail: zhining@bjtu.edu.cn;

Tel.: +86-10-51684663; Fax: +86-10-51688404.

Academic Editor: Kevin H. Knuth

Received: 5 November 2014 / Accepted: 12 January 2015 / Published: 30 January 2015

\begin{abstract}
A predictive model for droplet size and velocity distributions of a pressure swirl atomizer has been proposed based on the maximum entropy formalism (MEF). The constraint conditions of the MEF model include the conservation laws of mass, momentum, and energy. The effects of liquid swirling strength, Weber number, gas-to-liquid axial velocity ratio and gas-to-liquid density ratio on the droplet size and velocity distributions of a pressure swirl atomizer are investigated. Results show that model based on maximum entropy formalism works well to predict droplet size and velocity distributions under different spray conditions. Liquid swirling strength, Weber number, gas-to-liquid axial velocity ratio and gas-to-liquid density ratio have different effects on droplet size and velocity distributions of a pressure swirl atomizer.
\end{abstract}

Keywords: droplet size and velocity distributions; maximum entropy formalism; pressure swirl atomizers

\section{Introduction}

The liquid fuel injection process plays an important role in combustion performance, so the distributions of droplet size and velocity in sprays are crucial parameters needed for fundamental 
analysis of practical spray systems. Some spray applications require narrow size distributions, while some need wide ones. Other spray processes require very a few small drops or very few large ones [1]. Droplet velocity distribution affects further breakup of droplets. Droplet size and velocity distributions are typically described using one of the following four methods: empirical, maximum entropy formalism (MEF), discrete probability function method, or stochastic method [2-9]. Of the four available methods, former studies indicate that the MEF shows good prediction ability [10-15]. Due to its powerful prediction ability, the MEF has been used in many researches to study the size and velocity distribution characteristics in all kinds of spray systems [10-15].

Pressure swirl atomizers, which have good atomization characteristics, are very common parts of present combustion systems such as gas turbine and liquid-propellant rocket engines. Lots of research about pressure swirl atomizer spray characteristics has been done by experimental works, numerical simulation and theoretical analysis [16-20]. However, there are few reports about the prediction of droplet size or velocity distributions of pressure swirl atomizers [21,22]. For pressure swirl atomizers, based on maximum entropy formalism, only the effect of injection pressure on droplet size and velocity distributions has been reported [22].

The main objective of this paper is to predict and study droplet size and velocity distributions of a pressure swirl atomizer under different spray conditions. Joint probability density function of droplet size and velocity is determined by maximum entropy formalism. The constants in the joint probability density function are determined by the results of former linear and nonlinear instability analysis of an annular swirling viscous liquid sheet. Liquid swirling strength, Weber number, gas-to-liquid axial velocity ratio and gas-to-liquid density ratio are changed to study their effects on droplet size and velocity distributions of a pressure swirl atomizer.

\section{Mathematical Formulation}

In this paper, the maximum entropy formalism is applied to predict and study droplet size and velocity distributions of a pressure swirl atomizer spray under different injection conditions. The entropy was defined by Claude Shannon as follows [23]:

$$
S=-K \sum_{i=1}^{n} p_{i} \ln p_{i}
$$

where $S$ is Shannon entropy. $p_{i}$ is the probability of occurrence of the state $i$ and $K$ is the Boltzmann constant.

Shannon entropy rests on the hypothesis that correlations among different parts of the system are short ranged [24,25]. Figure 1 shows interface deformation of an annular swirling viscous liquid sheet issued from a pressure swirl atomizer. The maximum entropy formalism is used in the breakup range of the liquid sheet shown in Figure 1. The atomization of liquids is a process involving long range correlations, while the breakup range is short. Maximum entropy formalism was successfully adopted in the breakup range to get droplet size and velocity distributions of liquid jets and sheets [1,12-14]. In this study, maximum entropy formalism was adopted to get droplet size and velocity distributions of an annular swirling viscous liquid sheet issued from a pressure swirl atomizer. 


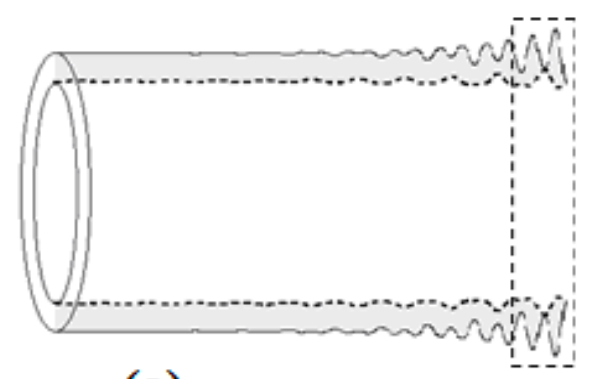

(a)

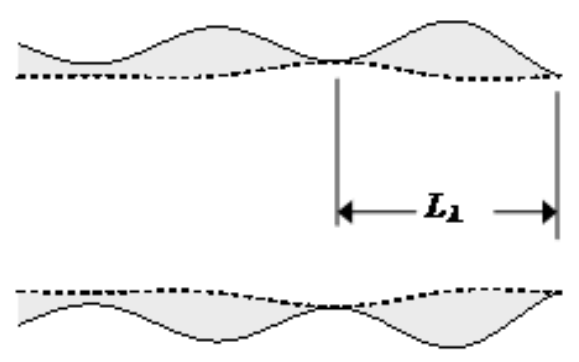

(b)

Figure 1. Interface deformation of an annular swirling viscous liquid sheet. (a) Liquid sheet interface development profile; (b) Local enlarged liquid sheet interface.

To apply governing equations and to determine droplet size and velocity distributions, a control volume is considered from the initial breakup point where the droplets form from ligaments. The droplet formation process in the control volume can be considered as a transformation from one to another equilibrium state. According to the thermodynamics laws, during a changing state the mass, momentum and energy are conserved while entropy maximization occurs [26]. The liquid sheet thickness and liquid axial velocity were used as characteristic length and characteristic velocity to obtain the dimensionless conservation equations. The dimensionless conservation equations for the liquid mass, momentum and energy can be written as follows:

$$
\begin{gathered}
\int_{D_{\min }}^{D_{\max }} \int_{U_{\min }}^{U_{\max }} f \bar{D}^{3} d \bar{U} d \bar{D}=1+\bar{S}_{m} \\
\int_{D_{\min }}^{D_{\max }} \int_{U_{\min }}^{U_{\max }} f \overline{U D}^{3} d \bar{U} d \bar{D}=1+\bar{S}_{m v} \\
\int_{D_{\min }}^{D_{\max }} \int_{U_{\min }}^{U_{\max }} f\left(\bar{D}^{3} \bar{U}^{2}+B \bar{D}^{2}\right) d \bar{U} d \bar{D}=1+\bar{S}_{e}
\end{gathered}
$$

where $f$ is joint probability density function of droplet size and velocity. $\bar{S}_{m}, \bar{S}_{m v}, \bar{S}_{e}$ denote the dimensionless mass, momentum and energy source terms, respectively. $\bar{D}$ and $\bar{U}$ are dimensionless droplet diameter and velocity, respectively. The parameter $B$ is a constant and is related to the surface tension through the following relation: $B=12 \sigma / \rho_{l} U_{l}^{2} D_{30}$ and $D_{30}$ is mass mean diameter of droplets.

In addition to Equations (2)-(4), according to the probability concept, total summation of probabilities should be equal to unity:

$$
\int_{D_{\min }}^{D_{\max }} \int_{U_{\min }}^{U_{\max }} f d \bar{U} d \bar{D}=1
$$

So the problem of determining the joint probability density function $f$ is turned into a constrained optimization problem as following: 


$$
\begin{array}{ll}
\max & S=-K \int_{D_{\min }}^{D_{\max }} \int_{U_{\min }}^{U_{\max }} f \ln f d \bar{U} d \bar{D} \\
\text { s.t. } & \int_{D_{\min }}^{D_{\max }} \int_{U_{\min }}^{U_{\max }} f \bar{D}^{3} d \bar{U} d \bar{D}=1+\bar{S}_{m} \\
& \int_{D_{\min }}^{D_{\max }} \int_{U_{\min }}^{U_{\max }} f \bar{D}^{3} \bar{U} d \bar{U} d \bar{D}=1+\bar{S}_{m v} \\
& \int_{D_{\min }}^{D_{\max }} \int_{U_{\min }}^{U_{\max }} f\left(\bar{D}^{3} \bar{U}^{2}+B \bar{D}^{2}\right) d \bar{U} d \bar{D}=1+\bar{S}_{e} \\
& \int_{D_{\min }}^{D_{\max }} \int_{U_{\min }}^{U_{\max }} f d \bar{U} d \bar{D}=1
\end{array}
$$

The source terms in Equation (6) are as follows:

$$
\begin{gathered}
\bar{S}_{m}=0 \\
\bar{S}_{m v}=Q\left(W-U_{l}^{*}\right)^{2} C_{D} \\
\bar{S}_{e}=\frac{1}{2} Q\left(W-U_{l}^{*}\right)^{3} S_{0} C_{D}
\end{gathered}
$$

where $U_{l}^{*}=\sqrt{1+\left(E\left(2 A_{h}+1\right) / 2\right)^{2}}, E$ is liquid swirling strength, $S=2 \pi\left(2 A_{h}+1\right) L_{b}, C_{D}$ is drag coefficient, $L_{b}$ is liquid sheet breakup length, $Q$ is gas-to-liquid density ratio, $W$ is gas-to-liquid velocity ratio, Breakup length $L_{b}$ is related to the atomizer injection conditions which are determined by Weber number, liquid swirling strength, gas-to-liquid density ratio, gas-to-liquid velocity ratio, et al. In the linear and nonlinear theory, the breakup length was computed [27,28]. Table 1 shows the relation between breakup length and liquid swirling strength.

Table 1. The breakup length of an annular swirling liquid sheet under different liquid swirling strengths.

\begin{tabular}{|l|l|l|l|l|l|l|}
\hline Dimensionless liquid swirling strength & 0 & 0.01 & 0.02 & 0.03 & 0.04 & 0.05 \\
\hline Dimensionless breakup length & 393 & 340 & 105 & 56 & 41 & 22 \\
\hline
\end{tabular}

Source terms were get under different parameters, including Weber number, etc. The Lagrange multipliers method was used to deal with Equations (6). The number-based joint probability density function $f$ for droplet size and velocity distributions is solved as Equation (7):

$$
f=f_{0} \exp \left[-1-\Lambda_{0}-\Lambda_{1} \bar{D}^{3}-\Lambda_{2} \bar{D}^{3} \bar{U}-\Lambda_{3}\left(\bar{D}^{3} \bar{U}^{2}+B \bar{D}^{2}\right)\right]
$$

where $f_{0}$ is a prior droplet size probability density function [14]. $\Lambda_{i}(i=0,1,2,3)$ are Lagrange multipliers to be determined by the maximum entropy formalism. Furthermore, the number-based droplet size distribution can be obtained by integrating Equation (7) over the velocity space from minimum to maximum value. Similarly, the number-based droplet velocity distribution can be obtained by integrating Equation (7) over the diameter space from minimum to maximum value. Hence, the number-based droplet size and velocity distributions can be expressed as:

$$
\begin{gathered}
\frac{\mathrm{d} N}{\mathrm{~d} \bar{D}}=\int_{U_{\min }}^{U_{\max }} f \mathrm{~d} \bar{U}=\frac{\sqrt{\pi} f_{0}}{2 \sqrt{\bar{D}^{3} \Lambda_{3}}} \exp \left[-1-\Lambda_{0}-\Lambda_{1} \bar{D}^{3}+\frac{\Lambda_{2}^{2}}{4 \Lambda_{3} \bar{D}}-\Lambda_{3} B \bar{D}^{2}\right]\left[\operatorname{erf}\left(X_{\max 1}\right)-\operatorname{erf}\left(X_{\min 1}\right)\right] \\
\frac{\mathrm{d} N}{\mathrm{~d} \bar{U}}=\int_{D_{\min }}^{D_{\max }} f \mathrm{~d} \bar{D}
\end{gathered}
$$


where $\operatorname{erf}(X)$ denotes the error function and:

$$
\begin{aligned}
& X_{\max 1}=\sqrt{\Lambda_{3} \bar{D}^{3}}\left(\bar{U}_{\max }+\frac{\Lambda_{2}}{2 \Lambda_{3} \bar{D}^{2}}\right) \\
& X_{\min 1}=\sqrt{\Lambda_{3} \bar{D}^{3}}\left(\bar{U}_{\min }+\frac{\Lambda_{2}}{2 \Lambda_{3} \bar{D}^{2}}\right)
\end{aligned}
$$

Equations (8) and (9) are solved numerically to obtain the droplet size and velocity distributions of a pressure swirl atomizer. In this study, the effects of different dimensionless numbers on droplet size and velocity distributions were studied with Equations (8) and (9). The transition to scaling in size distribution functions was demonstrated in $[29,30]$.

The minimum diameter and velocity of droplets could be set to 0 . And maximum diameter and velocity of droplets could be determined by following equations:

$$
\begin{aligned}
& \bar{D}_{\text {max }}=\frac{10}{\rho_{l} W e_{30}\left(\bar{U}_{j} \cos \theta-W\right)^{2}} \\
& \bar{U}_{\max }=\frac{1}{\cos \theta}\left(W+\sqrt{\frac{10}{Q W e_{30} \bar{D}_{i}}}\right)
\end{aligned}
$$

where $\bar{U}_{j}=U_{j} / U_{l}$ is the droplet dimensional velocity and $W e_{30}$ is the Weber number of the droplet which has average mass.

\section{Results and Discussion}

Joint probability density of droplet size and velocity is computed using Equation (7). Figure 2 shows a droplet size and velocity joint probability density distribution obtained with maximum entropy formalism.

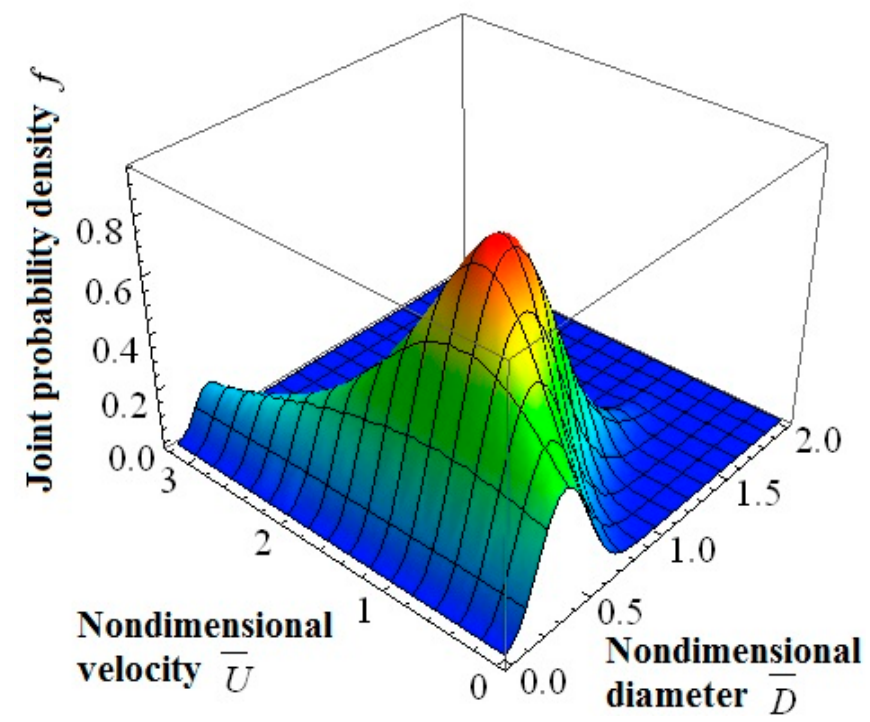

Figure 2. Joint probability density distribution of size and velocity of droplets in a spray issued from a pressure swirl atomizer. 
As is seen in Figure 2, the joint probability density distribution of size and velocity of droplets in the spray issued from a pressure swirl atomizer can be predicted with the maximum entropy formalism. Then, the droplet size distribution and droplet velocity distribution can be computed using Equations (8) and (9). The droplet size and velocity distributions computed in this paper will be validated and then a parametric variation of input conditions is made to study their effects on droplet size and velocity distributions.

\subsection{Results Validation}

Results of this study are first compared with experimental and calculation results of reference [14] as is shown in Figure 3. The comparison shows that the droplet size distribution and droplet velocity distribution of this paper accord well with the experiment results of reference [14]. The comparisons show that the model based on maximum entropy formalism can predict droplet size and velocity distributions well for a pressure swirl atomizer. The sheet thickness at the atomizer exit is $192 \mu \mathrm{m}$, the gas-to-liquid density ratio is $1.206 \times 10^{-3}$ and the liquid velocity is $4.2 \mathrm{~m} / \mathrm{s}$.

As can be seen in Figure 3a, the peak value of droplet size distribution accords well with the experimental value. While the corresponding droplet diameter to the peak value of droplet size has little deviation. As can be seen in Figure 3b, although the droplet velocity distribution does not accords as well as the droplet size distribution, the general shape of the calculation result agree with the experimental results basically. Table 2 shows quantitative comparisons of calculation results and experimental results. As also can be seen in Figure 3, calculation results of this study accord better than earlier calculation results of reference [14], especially for the droplet velocity distribution. The residual sums of squares between the calculation results and the experimental results in Figure $3 \mathrm{a}$ and $3 \mathrm{~b}$ are 1.08 and 1.66, respectively. However, for earlier calculations, those are 2.25 and 3.18, respectively.
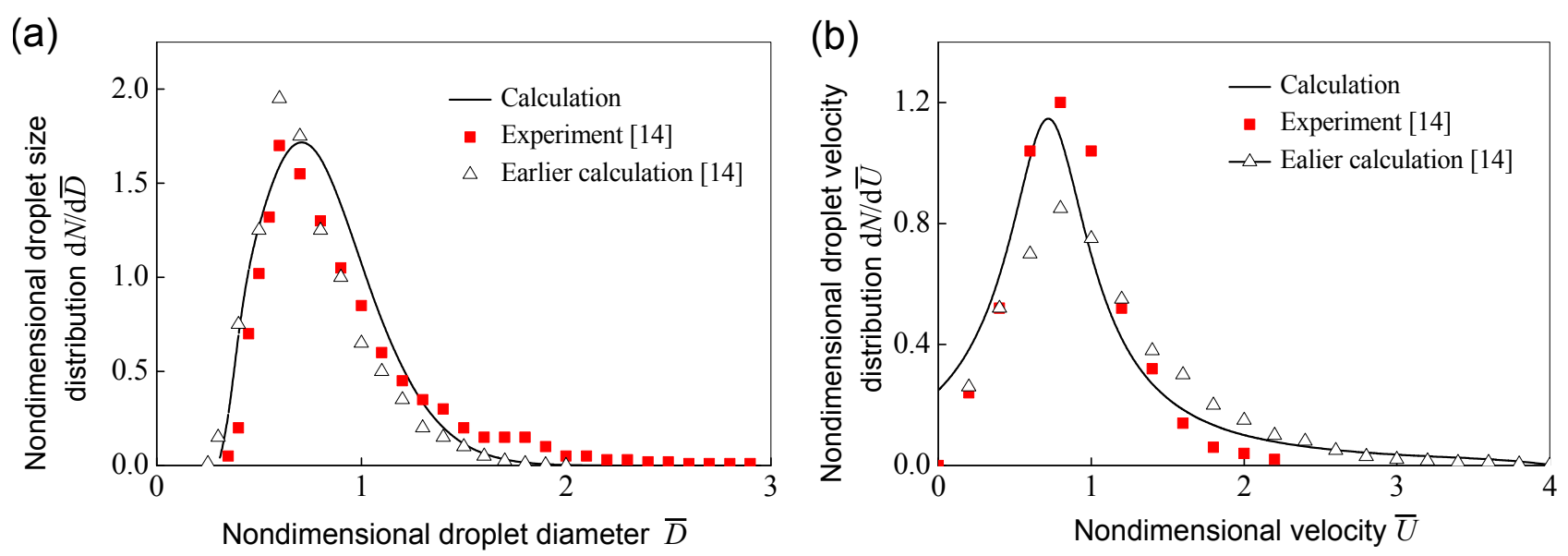

Figure 3. Results validation by comparison with dimensionless droplet size and velocity distributions of reference [14]. (a) Nondimensional droplet size distribution; (b) Nondimenaional droplet velocity distribution. 
Table 2. Quantitative comparisons of calculation results and experimental results of reference [14].

\begin{tabular}{cccccc}
\hline \multirow{2}{*}{ Results } & \multicolumn{2}{c}{ Droplet size distribution } & & \multicolumn{2}{c}{ Droplet velocity distribution } \\
\cline { 2 - 3 } & Calculation & Experiment & & Calculation & Experiment \\
\hline $\begin{array}{c}\text { Nondimensional droplet diameter } \\
\text { corresponds to peak value }\end{array}$ & 0.7 & 0.6 & & 0.72 & 0.8 \\
\hline Mean value & 0.78 & 0.72 & & 0.76 & 0.84 \\
\hline Standard deviation & 0.51 & 0.54 & & 0.55 & 0.52 \\
\hline
\end{tabular}

Liquid swirling strength is the major flow parameter of a pressure swirl atomizer. It has an important effect on instability and atomization of sprays of a pressure swirl atomizer which leads to different size and velocity distributions of breakup droplets. The liquid axial velocity, which is described by Weber number, is related to the aerodynamic effect and the shear force on the liquid surface. In air-blast liquid swirl atomizers, high speed axial air flow is used to improve the atomization effect of spray. The gas axial velocity, which is described by gas-to-liquid axial velocity ratio, is related to the axial velocity difference between liquid and gas also causes shear effect on liquid surface. High density gas environment is often used to improve the atomization effect of liquid sprays.

The droplet size and velocity distributions under different liquid swirling strength, Weber numbers, gas-to-liquid axial velocity ratios and gas-to-liquid density ratios will be studied.

\subsection{Effect of Liquid Swirling Strength}

Figure 4 shows the dimensionless droplet size distribution under different liquid swirling strength. The results show that as the liquid swirling strength is increased the mean droplet size of the spray increases first and then decreases and the distribution curve shifts towards a bigger diameter first and then shifts towards a smaller diameter. Furthermore, the maximum size of droplet decreases first and then increases. The results show that, for a determined pressure swirl atomizer liquid sheet, there is a critical liquid swirling strength value. The liquid swirling strength experiences a stabilizing effect on the liquid sheet breakup when the liquid swirling strength value is smaller than the critical liquid swirling strength value. While the liquid swirling strength experiences a destabilizing effect on the liquid sheet breakup when the liquid swirling strength value is higher than the critical liquid swirling strength value.

Figure 5 shows the dimensionless droplet velocity distribution under different liquid swirling strength. As can be seen in Figure 5, as the liquid swirling strength is increased, the mean droplet velocity of the spray increases and the distribution curve shifts towards a bigger velocity. Furthermore, the maximum velocity of droplet increases. Results show that the liquid swirling has effects on decreasing the number of small-velocity droplets and increasing the number of big-velocity droplets. The increase of droplet velocity is helpful to further breakup of droplets. 


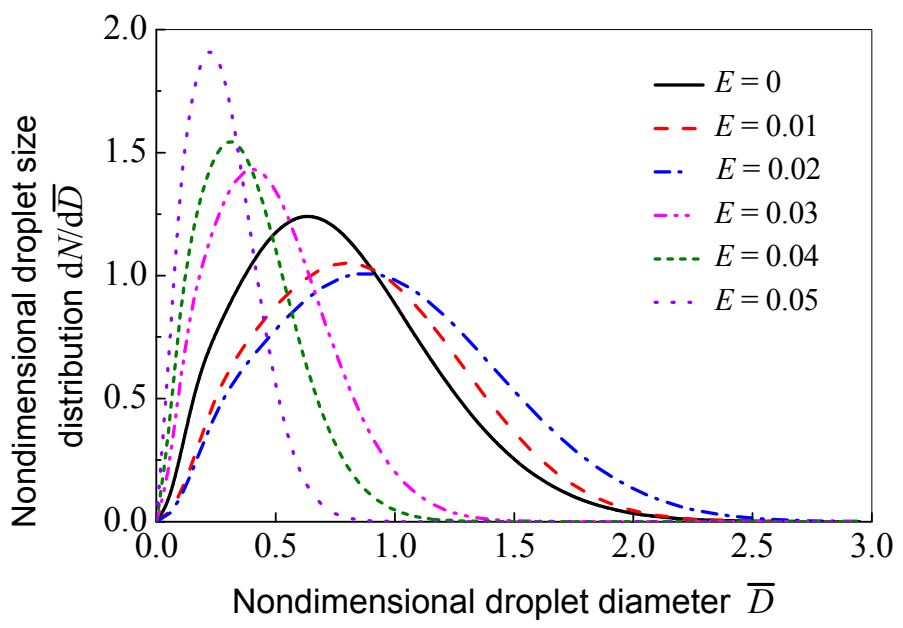

Figure 4. Number density of droplet diameter versus droplet dimensionless diameter under different liquid swirling strength. $W e=1000, R e=900, Q_{i}=Q_{o}=0.001, A_{h}=4, B_{h}=5$, $W_{i}=W_{o}=0$.

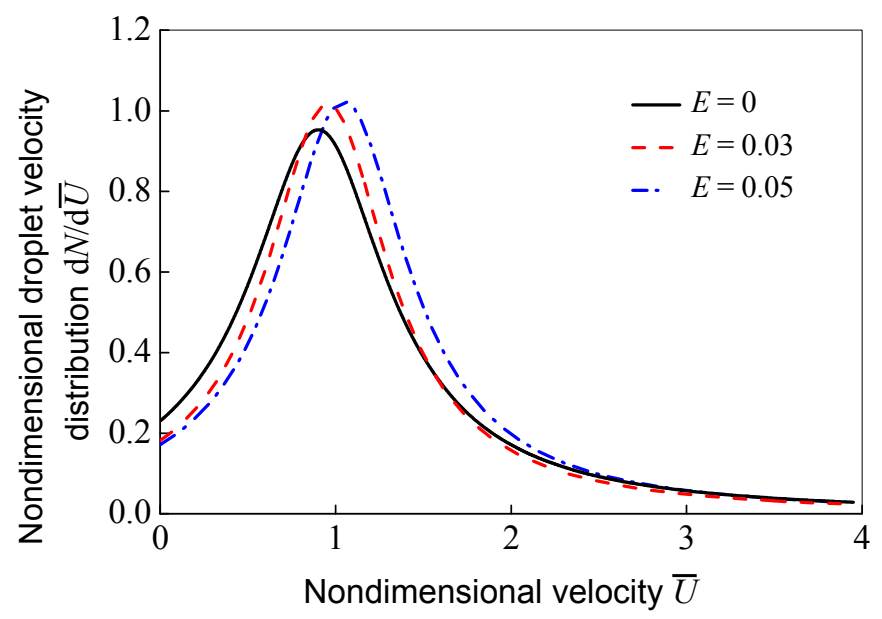

Figure 5. Number density of droplet velocity versus droplet dimensionless velocity under different liquid swirling strength. $W e=1000, R e=900, Q_{i}=Q_{o}=0.001, A_{h}=4, B_{h}=5$, $W_{i}=W_{o}=0$.

\subsection{Effect of Weber Number}

Liquid swirling velocity and liquid axial velocity are both liquid motion parameters. The effect of liquid axial velocity has a simpler effect on liquid droplet size distribution than liquid swirling velocity. The liquid axial velocity is characterized by Weber number. Figure 6 shows the dimensionless droplet size distribution under different Weber numbers. As can be seen in Figure 6, as the Weber number is increased the mean droplet size of the spray decreases and the distribution curve shifts towards a smaller diameter. Furthermore, the maximum size of droplet increases. Results show that by increasing the Weber number, the spray experiences a destabilizing effect. 


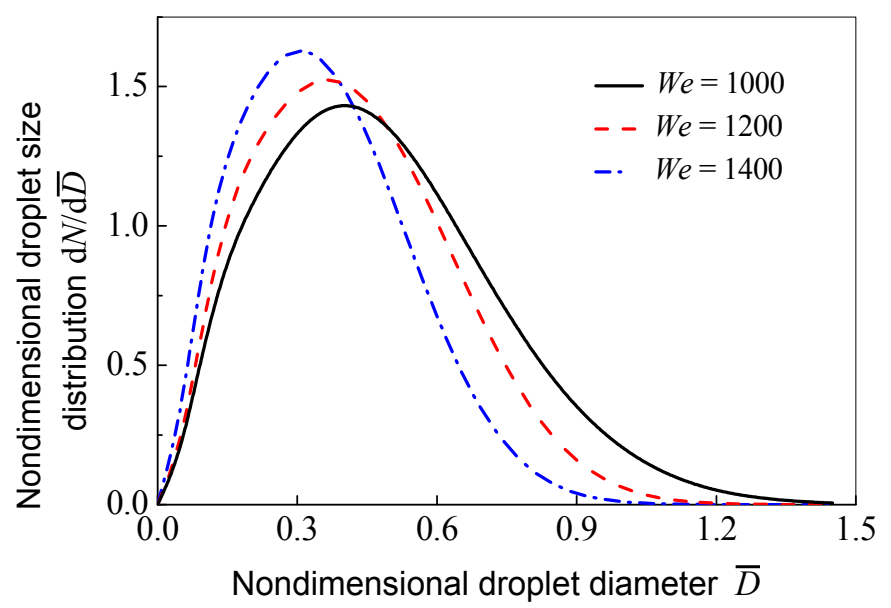

Figure 6. Number density of droplet diameter versus droplet dimensionless diameter under different Weber numbers. $R e=900, E=0.03, Q_{i}=Q_{o}=0.001, A_{h}=4, B_{h}=5, W_{i}=W_{o}=0$.

Figure 7 shows the dimensionless droplet velocity distribution under different Weber numbers. As the Weber number is increased the mean droplet velocity of the spray increases slightly and the distribution curve moves close to the mean velocity. Furthermore, the maximum velocity of droplet increases. Results show that the increase of liquid axial velocity has slightly promoting effect on further breakup of droplets.

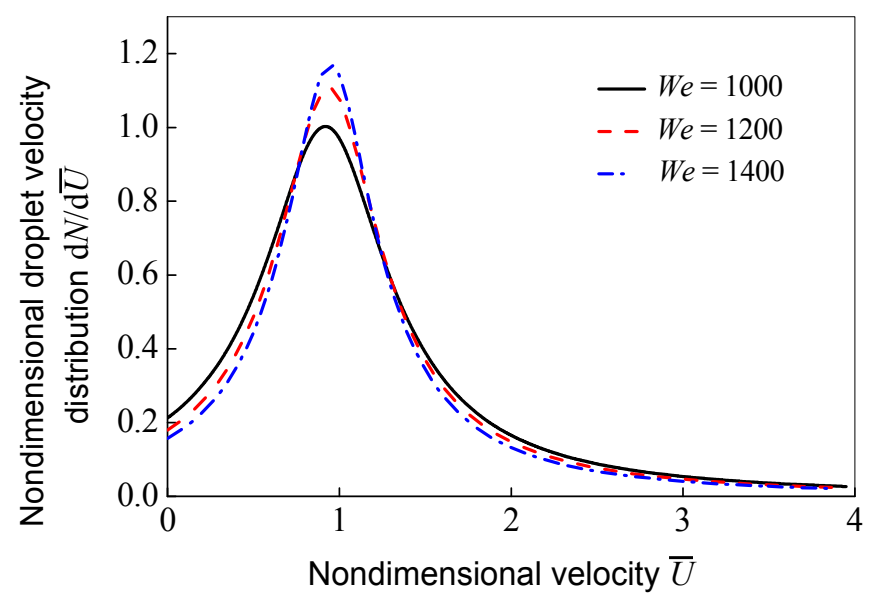

Figure 7. Number density of droplet velocity versus droplet dimensionless velocity under different Weber numbers. $R e=900, E=0.03, Q_{i}=Q_{o}=0.001, A_{h}=4, B h=5, W_{i}=W_{o}=0$.

\subsection{Effect of Gas-to-Liquid Axial Velocity Ratio}

The breakup of liquid sheet is a result of shear effect between liquid and surrounding gas. The gas motion has an important effect on liquid droplet size and velocity distributions. The gas motion is characterized by gas-to-liquid axial velocity ratio. Figure 8 shows the dimensionless droplet size distribution under different gas-to-liquid axial velocity ratios. As can be seen in Figure 8, as the gas-to-liquid axial velocity ratio is increased the mean droplet size of the spray increases first and then decreases and the distribution curve shifts towards a bigger diameter first and then shifts towards a small diameter. Furthermore, the maximum size of droplet decreases first and then increases. The axial velocity 
difference between gas and liquid causes the shear on the liquid surface. The bigger the axial velocity difference between gas and liquid, the more unstable the liquid spray and the smaller the droplet size. High speed gas flow is used in some kinds of atomizers, such as air-blast atomizers, to improve the atomization effect.

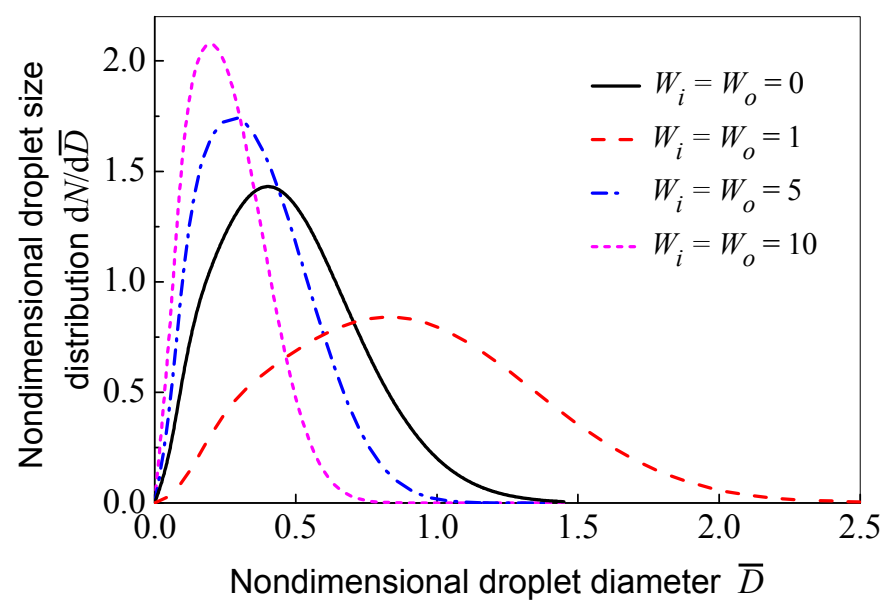

Figure 8. Number density of droplet diameter versus droplet dimensionless diameter under different gas-to-liquid axial velocity ratios. $W e=1000, R e=900, E=0.03, Q_{i}=Q_{o}=0.001$, $A_{h}=4, B_{h}=5$.

Figure 9 shows the dimensionless droplet velocity distribution under different gas-to-liquid axial velocity ratios. As can be seen in Figure 9, as the gas-to-liquid axial velocity ratio is increased the mean droplet velocity of the spray increases slightly and the distribution curve shifts towards a bigger velocity. The maximum velocity of droplet does not change much. Results show that bigger gas-to-liquid axial velocity ratio is helpful to further breakup of liquid droplets.

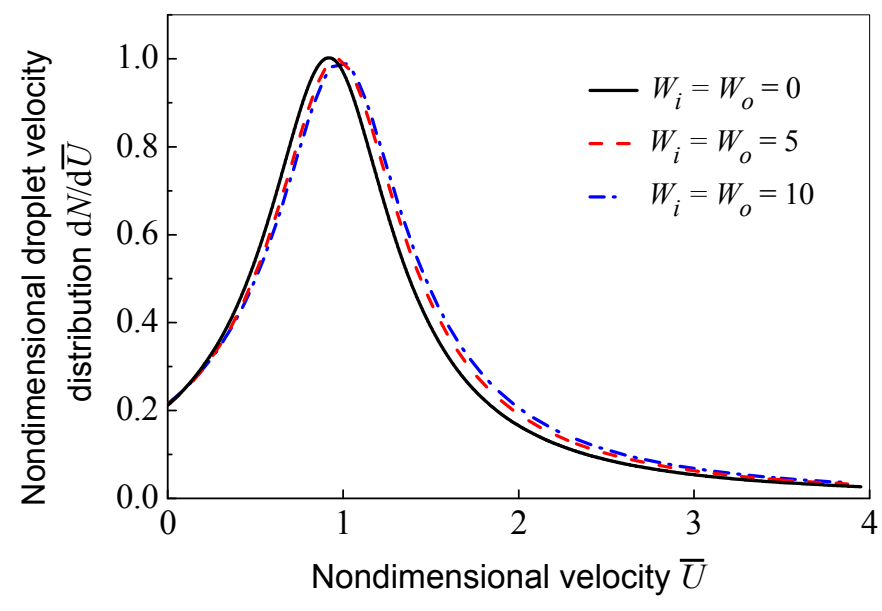

Figure 9. Number density of droplet velocity versus droplet dimensionless velocity under different gas-to-liquid axial velocity ratios. $W e=1000, R e=900, E=0.03, Q_{i}=Q_{o}=0.001$, $A_{h}=4, B_{h}=5$. 


\subsection{Effect of Gas-to-Liquid Density Ratio}

In practical project applications, the ambient gas where the liquid spray is injected into often has different densities. The ambient gas density has an important effect on breakup droplet size and velocity distributions. Here the effect of ambient gas density is studied by dimensionless number gas-to-liquid density ratio. Figure 10 shows the dimensionless droplet size distribution under different gas-to-liquid density ratios. As can be seen in Figure 10, as the gas-to-liquid density is increased the mean droplet size of the spray decreases and the distribution curve shifts towards a smaller diameter. Furthermore, the maximum size of droplet increases. Results show that by increasing the gas-to-liquid density ratio, the spray experiences a destabilizing effect and smaller droplets are generated.

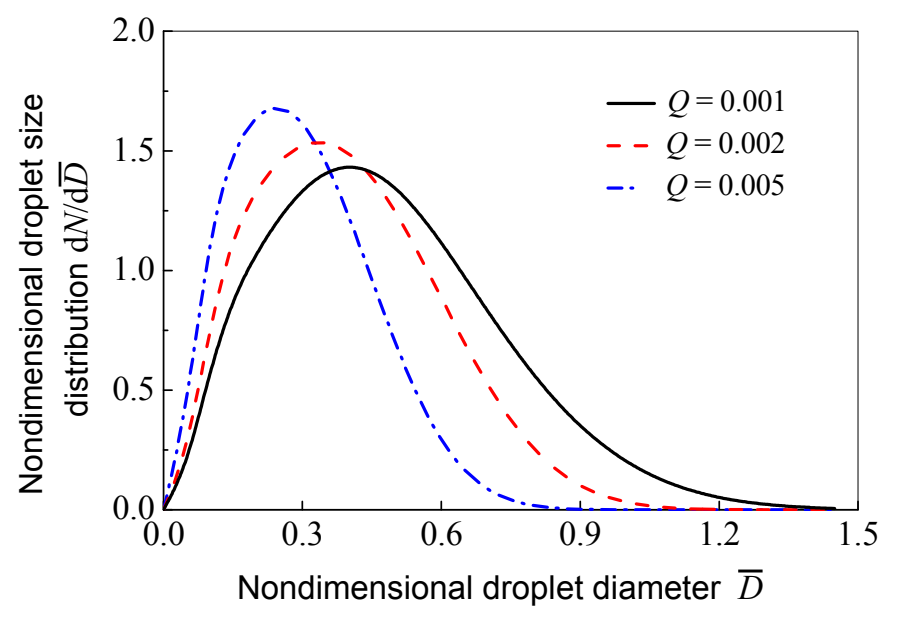

Figure 10. Number density of droplet diameter versus droplet dimensionless diameter under different gas-to-liquid density ratios. $W e=1000, R e=900, E=0.03, A h=4, B_{h}=5$, $W_{i}=W_{o}=0$.

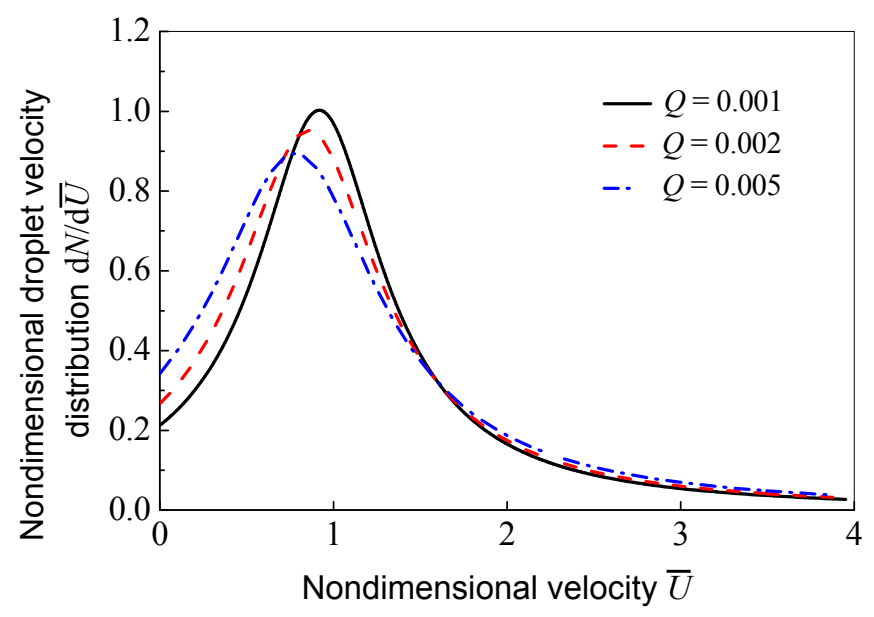

Figure 11. Number density of droplet velocity versus droplet dimensionless velocity under different gas-to-liquid density ratios. $W e=1000, R e=900, E=0.03, A_{h}=4, B_{h}=5$, $W_{i}=W_{o}=0$.

Figure 11 shows the dimensionless droplet velocity distribution under different gas-to-liquid density ratios. As can be seen in Figure 11, as the gas-to-liquid density ratio is increased the mean droplet 
velocity of the spray decreases and the distribution curve spreads more over the velocity space. Further, the maximum velocity of droplet decreases. Results show that the increase of gas density is helpful to improve the atomization effect of an annular swirling viscous liquid sheet from both droplet size distributions and velocity distribution views.

\section{Summary and Conclusions}

A predictive model for droplet size and velocity distributions of a pressure swirl atomizer has been proposed based on the MEF. The effects of liquid swirling velocity, liquid axial velocity, surrounding gas axial velocity and gas density on the droplet size and velocity distributions of a pressure swirl atomizer have been investigated with dimensionless numbers such as dimensionless liquid swirling strength, Weber number, gas-to-liquid axial velocity ratio and gas-to-liquid density ratio.

It is found that smaller average sizes and smaller distribution ranges of breakup droplets are obtained at higher liquid swirling strength, higher Weber numbers, higher gas-to-liquid axial velocity ratios or higher gas-to-liquid density ratios. Furthermore, bigger average velocity values are obtained at higher liquid swirling strength, higher gas-to-liquid axial velocity ratios or lower gas-to-liquid density ratios, while Weber number affects the average velocity value of breakup droplets only slightly.

\section{Acknowledgments}

The authors acknowledge the support provided by National Natural Science Foundation of China (51276011), Beijing Natural Science Foundation (3132016), National High Technology Research and Development Program of China (2013AA065303) and Opening Foundation of State Key Laboratory of Engines of China (k2013-3).

\section{Author Contributions}

Zhi Ning designed research; Kai Yan, Ming Lü and Chunhua Sun performed research and analyzed the data; Kai Yan wrote the paper. All authors have read and approved the final manuscript.

\section{Conflicts of Interest}

The authors declare no conflict of interest.

\section{References}

1. Ashgriz, N. Handbook of Atomization and Sprays: Theory and Applications; Springer-Verlag: New York, NY, USA, 2011.

2. Crow, E.L.; Shimizu, K. Lognormal Distributions: Theory and Applications; Marcel Dekker: New York, NY, USA, 1988.

3. Mugele, R.A.; Evans, H.D. Droplet size distributions in sprays. Ind. Eng. Chem. 1951, 43, 1317-1324.

4. Tate, R.W.; Marshall, W.R., Jr. Atomization by centrifugal pressure nozzles. Chem. Eng. Prog. 1953, 49, 169-174.

5. Rosin, P.; Rammler, E. The laws governing the fineness of powdered coal. J. Inst. Fuel 1933, 7, 29-36. 
6. Nukiyama, S.; Tanasawa, Y. Experiments on the atomization of liquids in an air stream reports 3: On the droplet-size distribution in an atomized jet. Trans. Jpn. Soc. Mech. Eng. 1939, 5, 62-67.

7. Bhatia, J.C.; Dominick, J.; Durst, F. Phase-Doppler-anemometry and the log-hyperbolic distribution applied to liquid sprays. Part. Part. Syst. Charact. 1988, 5, 153-164.

8. Bhatia, J.C.; Durst, F. Comparative study of some probability distributions applied to liquid sprays. Part. Part. Syst. Charact. 1989, 6, 151-162.

9. Bhatia, J.C.; Durst, F. Description of sprays using joint hyperbolic distribution in particle size and velocity. Combust. Flame 1990, 81, 203-218.

10. Xu, T.H.; Durst, F.; Tropea, C. The three-parameter log-hyperbolic distribution and its application to particle sizing. In Proceedings of the Fifth International Conference on Liquid Atomization and Spray Systems (ICLASS), Gaithersburg, MD, USA, 15-18 July 1991.

11. Sellens, R.W.; Brzustowski, T.A. A prediction of the drop size distribution in a spray from first principle. Atom. Spray Technol. 1985, 1, 89-102.

12. Li, X.; Tankin, R.S. Droplet size distribution: A derivation of a Nukiyama-Tanasawa type distribution function. Combust. Sci. Technol. 1987, 56, 65-76.

13. Mondal, D.; Datta, A.; Sarkar, A. Droplet size and velocity distributions in a spray from a pressure swirl atomizer: Application of maximum entropy formalism. J. Mech. Eng. Sci. 2004, 218, 737-749.

14. Kim, W.T.; Mitra, S.K.; Li, X.; Prociw, L.A.; Hu, T.C.J. A predictive model for the initial droplet size and velocity distributions in sprays and comparison with experiments. Part. Part. Syst. Charact. 2003, 20, 135-149.

15. Nath, S.; Datta, A.; Nukhopadhyay, A.; Sen, S.; Tharakan, T.J. Prediction of size and velocity distributions in sprays formed by the breakup of planar liquid sheets using maximum entropy formalism. Atom. Sprays 2011, 21, 483-501.

16. Chu, C.C.; Chou, S.F.; Lin, H.I.; Liann, Y.H. An experimental investigation of swirl atomizer sprays. Heat Mass Transf. 2008, 45, 11-22.

17. Siamas, G.A.; Jiang, X.; Wrobel, L.C. Numerical investigation of a perturbed swirling annular two-phase jet. Int. J. Multiph. Flow 2009, 30, 481-493.

18. Santangelo, P.E. Experiments and modeling of discharge characteristics in water-mist sprays generated by pressure-swirl atomizers. J. Therm. Sci. 2012, 21, 539-548.

19. Park, H.; Heister, S.D. Nonlinear simulation of free surfaces and atomization in pressure swirl atomizers. Phys. Fluids 2006, 18, doi:10.1063/1.2197876.

20. Chryssakis, C.A.; Assanis, D.N.; Lee, J.K.; Nishida, K. Fuel Spray Simulation of High-Pressure Swirl-Injector for DISI Engines and Comparison with Laser Diagnostic Measurements; SAE Technical Paper, No. 2003-01-0007; SAE: Warrendale, PA, USA, 2003.

21. Cousin, J.; Yoon, S.J.; Dumouchel, C. Coupling of classical linear theory and maximum entropy formalism for prediction of drop size distribution in sprays: Application to pressure-swirl atomizers. Atom. Sprays 1996, 6, 601-622.

22. Mondal, D.; Datta, A.; Sarkar, A. Droplet size and velocity distributions in a spray from a pressure swirl atomizer: Application of maximum entropy formalism. J. Mech. Eng. Sci. 2004, 218, 737-749.

23. Shannon, C.E. A mathematical theory of communications. Bell Labs Tech. J. 1948, 27, 379-423.

24. Sotolongo-Costa, O.; Rodrigues, A.H.; Rodgers, G.J. Tsallis entropy and the transition to scaling in fragmentation. Entropy 2000, 2, 172-177. 
25. Sotolongo-Costa, O. Scaling in fragmentation phenomena. In Proceedings of the 2nd International Conference on Physics and Industrial Development: Bridging the Gap (ICPID-II), Belo Horizonte, Brazil, 7-10 July 1996; pp. 293-297.

26. Movahednejad, E.; Ommi, F.; Hosseinalipour, S.M. Prediction of droplet size and velocity distribution in droplet formation region of liquid spray. Entropy 2010, 12, 1484-1498.

27. Yan, K.; Ning, Z.; Lü, M. Spatial instability analysis of an annular swirling viscous liquid jet. Chin. J. Theor. Appl. Mech. 2012, 44, 687-693.

28. Yan, K.; Jog, M.A.; Ning, Z. Nonlinear spatial instability of an annular swirling viscous liquid sheet. Acta Mech. 2013, 224, 3071-3090.

29. Sotolongo-Costa, O.; Moreno-Vega, Y.; Lloveras-González, J.J.; Antoranz, J.C. Criticallity in droplet fragmentation. Phys. Rev. Lett. 1996, 76, 42-46.

30. Ishii, T.; Matsusita, M. Fragmentation of long thin glass rods. J. Phys. Soc. Jpn. 1992, 61, 3474-3477.

(C) 2015 by the authors; licensee MDPI, Basel, Switzerland. This article is an open access article distributed under the terms and conditions of the Creative Commons Attribution license (http://creativecommons.org/licenses/by/4.0/). 